\title{
A Dopaminergic Axon Lattice in the Striatum and Its Relationship with Cortical and Thalamic Terminals
}

\author{
Jonathan Moss and J. Paul Bolam \\ Medical Research Council Anatomical Neuropharmacology Unit, Department of Pharmacology, University of Oxford, Oxford OX1 3TH, United Kingdom
}

\begin{abstract}
Interactions between glutamatergic corticostriatal afferents and dopaminergic nigrostriatal afferents are central to basal ganglia function. The thalamostriatal projection provides a glutamatergic innervation of similar magnitude to the corticostriatal projection. We tested the hypotheses that (1) thalamostriatal synapses have similar spatial relationships with dopaminergic axons as corticostriatal synapses do and (2) the spatial relationships between excitatory synapses and dopaminergic axons are selective associations. We examined at the electron microscopic level rat striatum immunolabeled to reveal vesicular glutamate transporters (VGluTs) 1 and 2, markers of corticostriatal and thalamostriatal terminals, respectively, together with tyrosine hydroxylase (TH) to reveal dopaminergic axons. Over $80 \%$ of VGluT-positive synapses were within $1 \mu \mathrm{m}$ of a TH-positive axon and $>40 \%$ were within $1 \mu \mathrm{m}$ of a TH-positive synapse. Of structures postsynaptic to VGluT1- or VGluT2-positive terminals, 21 and 27\%, respectively, were apposed by a TH-positive axon and about half of these made synaptic contact. When structures postsynaptic to VGluT-positive terminals and VGluT-positive terminals themselves were normalized for length of plasma membrane, the probability of them being apposed by, or in synaptic contact with, a TH-positive axon was similar to that of randomly selected structures. Extrapolation of the experimental data to more closely reflect the distribution in 3D reveals that all structures in the striatum are within $\sim 1 \mu \mathrm{m}$ of a TH-positive synapse. We conclude that (1) thalamostriatal synapses are in a position to be influenced by released dopamine to a similar degree as corticostriatal synapses are and (2) these associations arise from a nonselective dopaminergic axon lattice.
\end{abstract}

Key words: basal ganglia; nigrostriatal; synapses; microcircuits; thalamostriatal; corticostriatal; dopamine-glutamate interactions

\section{Introduction}

The basal ganglia are a group of subcortical nuclei that are involved in a variety of functions including motor, cognitive and mnemonic behaviors (DeLong, 1990; Graybiel, 1995, 2000; Gerfen and Wilson, 1996; Bolam et al., 2000). Central to our understanding of basal ganglia function is the relationship between the glutamatergic projection from the cortex to the principal division of the basal ganglia, the striatum, and the dopaminergic innervation of the same region, derived from the substantia nigra pars compacta (Kemp and Powell, 1971; Wilson et al., 1983; Bouyer et al., 1984; Freund et al., 1984). Excitatory corticostriatal afferents mainly innervate the spines of medium-sized spiny projection neurons (Frotscher et al., 1981; Somogyi et al., 1981; Smith et al., 1998) and the response of the postsynaptic structure is modulated by synaptically released dopamine (Kerr and Wickens, 2001; Reynolds et al., 2001; Surmeier et al., 2007; Shen et al., 2008). Furthermore, the modulatory effect of dopamine on cortical transmission may also occur by synaptic spill-over and/or nonsynaptic release (Gonon, 1997; Cragg and Rice, 2004; Rice

Received June 18, 2008; revised Sept. 9, 2008; accepted Sept. 17, 2008

This work was supported by the Medical Research Council, UK. J.M. was supported by a Medical Research Council studentship. We thank Caroline Francis, Liz Norman, Katie Whitworth, and Ben Micklem for technical support and Justin Boyes for help in the initial stages of this project. We also thank Matt Brown, Pablo Henny, Pete Magill, Francis Marriott, Juan Mena-Segovia, and Jozsef Somogyi for helpful discussions and for comments on this manuscript.

Correspondence should be addressed to J. Paul Bolam, Medical Research Council Anatomical Neuropharmacology Unit, Department of Pharmacology, Mansfield Road, 0xford 0X13TH, UK. E-mail: paul.bolam@pharm.ox.ac.uk. D01:10.1523/JNEUROSCI.2780-08.2008

Copyright $\odot 2008$ Society for Neuroscience ～0270-6474/08/2811221-10\$15.00/0 and Cragg, 2008) acting presynaptically and/or postsynaptically. The molecular mechanisms underlying the modulatory role of dopamine are numerous and dependent on a variety of factors including the type of dopamine receptor; the outcome is a facilitation or attenuation of the excitatory transmission (for review, see Surmeier et al., 2007).

In addition to the excitatory input from the cortex, the striatum also receives a major glutamatergic projection from the thalamus, principally arising from the intralaminar nuclei (Xu et al., 1991; Groenewegen and Berendse, 1994; Smith et al., 2004; Raju et al., 2006, 2008). Although tracing studies have given some indication about the density of the thalamostriatal projection, the discovery that the VGluTs 1 and 2 are markers of cortical and thalamic terminals, respectively (Fremeau et al., 2001; Herzog et al., 2001; Kaneko and Fujiyama, 2002; Fujiyama et al., 2004, 2006), has led to the finding that the number of thalamostriatal terminals is of a similar magnitude to the number of corticostriatal terminals (Lacey et al., 2005). The question arises, therefore, whether glutamatergic thalamostriatal synapses have the same spatial relationship with dopaminergic terminals as has been proposed for corticostriatal synapses and are thus in a position to be equally modulated by released dopamine (Smith et al., 1994; Pinto et al., 2003). The main aim of the present study was to test this hypothesis. Since the dopaminergic nigrostriatal projection is remarkably dense (Björklund and Lindvall, 1984), the second aim was to test the hypothesis that the spatial relationships between glutamatergic synapses and dopaminergic axons represent 
selective associations and are not random in nature. To achieve these aims, we performed a quantitative analysis at the electron microscopic level of rat striatum immunolabeled to reveal cortical or thalamic terminals, using antibodies against VGluT1 or 2, together with antibodies against $\mathrm{TH}$ as a marker of dopaminergic axons.

\section{Materials and Methods}

Experimental procedures were performed on twelve adult male Sprague Dawley rats (245-425 g; Charles River), and were conducted in accordance with the Animals (Scientific Procedures) Act 1986 (UK), and the Society for Neuroscience policy on the use of animals in research. Pilot and control experiments were conducted on six of the rats to establish the optimal staining conditions and the detailed quantitative analysis on the remaining six.

Tissue preparation. The rats were anesthetized with sodium pentobarbitone ( $200 \mathrm{mg} / \mathrm{kg}$; Rhône Mérieux) then perfused via the aorta with $\sim 50$ $\mathrm{ml}$ of phosphate buffered saline (PBS; $0.01 \mathrm{~m}$ phosphate buffer, $\mathrm{pH} 7.4$, $0.876 \% \mathrm{NaCl}, 0.02 \% \mathrm{KCl})$ followed by $200 \mathrm{ml}$ of fixative $(0.1 \mathrm{M}$ phosphate buffer, $\mathrm{pH} 7.4,3 \%$ paraformaldehyde, $0.1 \%$ glutaraldehyde) over $\sim 25$ min. Free fixative was removed by postperfusion with PBS. The brain was removed and cut into $65 \mu \mathrm{m}$ sagittal sections ( 1 in 6 series) using a vibrating blade microtome (VT1000S; Leica).

Immunohistochemistry. All sections were washed five times in PBS and placed into a cryoprotectant ( $0.05 \mathrm{M}$ phosphate buffer, $25 \%$ sucrose, $10 \%$ glycerol) for a minimum of $2 \mathrm{~h}$ before freeze-thawing. Each section was then immersed in chilled isopentane and then liquid nitrogen for a few seconds, left to thaw at $\sim 25^{\circ} \mathrm{C}$ for $\sim 5 \mathrm{~min}$ and then washed three times in PBS. The sections were then double-immunolabeled to reveal VGluT1 or VGluT2, as markers of cortical and thalamic terminals, respectively, and to reveal tyrosine hydroxylase $(\mathrm{TH}$; rate limiting enzyme in the synthesis of catecholamines) as a marker of dopaminergic axons and terminals. Although TH immunolabeling will also reveal noradrenergic axons, these are rare in the striatum (Carlsson, 1959; Moore and Card, 1984). Normal goat serum in PBS (NGS-PBS; Vector Laboratories) was used to block (10\% NGS) and wash (2\% NGS) sections before the addition of primary antibodies. Primary antibodies for each of the doubleimmunolabeling experiments were added sequentially. Antibodies against VGluT1 or VGluT2 raised in rabbits (VGluT1, Mab Technologies; VGluT2, Synaptic Systems) were used at a dilution of 1:2000 in 2\% NGS-PBS and the sections were incubated overnight ( $15 \mathrm{~h}$ ), shaking at room temperature or over three nights $(64 \mathrm{~h})$ at $4^{\circ} \mathrm{C}$. They were then washed once in 2\% NGS-PBS, three times in PBS and once in 1\% NGSPBS. The sections were then incubated for $2 \mathrm{~h}$ in goat anti-rabbit goldconjugated antibody ( $1.4 \mathrm{~nm}$ colloidal gold, Nanoprobes) at a dilution of 1:100 in 1\% NGS-PBS. This was followed by one wash in 1\% NGS-PBS and three washes each in PBS and acetate buffer ( $0.1 \mathrm{~m}$ sodium acetate 3-hydrate) in preparation for silver intensification of the conjugated gold particles. Silver reagent ( $1 \mathrm{ml}$; HQ Silver kit, Nanoprobes) was added to each section and allowed to react for 2-4 min, washed three times each in acetate buffer and PBS, and once in 2\% NGS-PBS. They were then incubated in a mouse monoclonal antibody raised against TH (Sigma) at a dilution of 1:1000 in 2\% NGS-PBS overnight, shaking at room temperature. Washes of once $2 \%$ NGS-PBS, three times PBS and once $1 \%$ NGSPBS preceded the incubation in biotin-conjugated goat anti-mouse secondary antibody (BA9200, Vector Laboratories; diluted 1:200 in 1\% NGS-PBS). They were incubated with shaking at room temperature, for a minimum of $2 \mathrm{~h}$ before one wash in 1\% NGS-PBS, three washes of PBS and then incubated in avidin-biotin-peroxidase complex (ABC; Vector Laboratories, prepared according to the manufacturers instructions) with shaking for $90 \mathrm{~min}$ at $\sim 25^{\circ} \mathrm{C}$.

After the ABC incubation the sections were washed three times in PBS and twice in Tris buffer (0.05 M, pH 7.4). Diaminobenzidine (DAB; 2.5 $\mathrm{ml}$ of $0.025 \%$ in Tris buffer; Sigma) was added to the sections with regular mixing for 15 min then $40 \mu \mathrm{l}$ of $\mathrm{H}_{2} \mathrm{O}_{2}\left(0.03 \%\right.$ in $\left.\mathrm{H}_{2} \mathrm{O}\right)$ was added. The reaction continued for 5-7 min until staining was revealed and the reaction was stopped by the addition of Tris buffer before two further washes in Tris buffer and three washes in PBS. The sections were then washed into $0.1 \mathrm{M}$ phosphate buffer (PB; $\mathrm{pH}$ 7.4).

The primary antibodies were raised against rat VGluT1, VGluT2 and TH (amino acids 543-560, 510-582 and 40-152, respectively). The distribution of immunolabeling at the light and electron microscopic levels was distinct for each primary antibody, and consistent with previous observations by ourselves (TH, Magill et al., 2001; VGluTs 1 and 2, Lacey et al., 2005) and others (same antibodies: TH, Prasad and Amara, 2001; VGluT1, Villalba et al., 2006; VGluT2, Härtig et al., 2003. Different antibodies: VGluTs 1 and 2, Fremeau et al., 2001; Herzog et al., 2001; Kaneko and Fujiyama, 2002; Fujiyama et al., 2004, 2006). No immunolabeling was observed after omission of the primary antibodies and omission of each of the secondary antibodies individually showed no evidence of cross-reactivity.

The sections were then placed flat on the bottom of glass Petri dishes and postfixed in osmium tetroxide (1\% in PB; Oxkem) for 7 min. They were then washed in $0.1 \mathrm{M} \mathrm{PB}$ and dehydrated in an ascending series of ethanol dilutions (15 min in 50\% ethanol, $35 \mathrm{~min}$ in 70\% ethanol which included $1 \%$ uranyl acetate; TAAB; 15 min in 95\% ethanol, and twice 15 min in absolute ethanol). After absolute ethanol, sections were exposed to two changes of propylene oxide (Sigma) for $15 \mathrm{~min}$ and lifted, using modified forceps, into resin (Durcupan ACM, Fluka) in crafted foil boats and left overnight $(15 \mathrm{~h})$ at room temperature. The resin was then warmed to decrease its viscosity and sections were placed on microscope slides, a coverslip applied and the resin cured at $65^{\circ} \mathrm{C}$ for $\sim 70 \mathrm{~h}$.

Electron microscopic analysis. All sections were examined in the light microscope and areas from the dorsolateral striatum were cut from the slide, glued to the top of a resin block and trimmed with razor blades. Serial sections, $\sim 50 \mathrm{~nm}$ thick (silver/gray), were then cut using an ultramicrotome (Leica EM UC6) and were collected on pioloform-coated, single-slot copper grids (Agar Scientific). The sections were then leadstained to improve contrast for electron microscopic examination. A Philips CM10 electron microscope was used to examine stained tissue. Analyses were performed at a minimum of $5 \mu \mathrm{m}$ from the tissue-resin border (i.e., the surface of the section). The maximum distance from the tissue-resin border examined was determined by the penetration of the gold conjugated antibody together with the angle at which the tissueresin was sectioned, and was therefore variable. VGluT-immunopositive synapses were selected at random in the following manner: at a magnification of approximately $\times 6,200$ (at which synapses cannot be seen), a point was selected and centered, the magnification was increased to approximately $\times 25,000$ and the first synapse-forming VGluT1- or VGluT2-immunopositive terminal encountered was selected for analysis. Subsequently, any other immunopositive terminals seen within the frame were also analyzed. Any immunopositive terminals not fully within the frame were discarded. Postsynaptic structures, such as large dendrites that extended beyond the borders of the frame, were only examined within the frame. It should be noted that this may lead to an underestimation of the frequency of occurrence of relationships between glutamatergic and dopaminergic terminals. The criterion for an immunopositive structure was two or more silver intensified immunogold particles (hereafter simply referred to as immunogold particles). Images of the labeled terminals were digitally recorded (Gatan multiscan CCD camera, Gatan) in each of the six serial sections (final magnification in digital micrographs of $\times 54,100-99,100)$. In these images, we characterized the postsynaptic target(s) of the VGluT-positive terminals. Dendritic shafts were identified by their relatively large size when compared with spines, the presence of mitochondria and, although not essential, the protrusion of spines from their shaft. Spines were identified by their shape and relatively small size when compared with dendritic shafts, the lack of mitochondria within their cytoplasm and, although not essential, the presence of spiny apparatus. Cell bodies were identified by their relatively larger cytoplasmic space when compared with dendritic shafts, the presence of multiple intracellular compartments and organelles and, although not essential, the protrusion of large proximal dendrites from their membrane. Once identified, the relationships of these structures, and the VGluT-positive terminals themselves, with TH-immunopositive axons and synapses were examined. A total of 50 immunopositive terminals from each of three animals for both VGluT1/TH and VGluT2/TH 
immunostained tissue were identified in this way (total number of terminals $=300$ ). Analyses were performed on the digital images using the publicly available software, ImageJ (rsb.info.nih.gov/ij), and they were adjusted for contrast and brightness using Adobe Illustrator and Photoshop (Version CS2, Adobe). We recorded the frequency of appositions and synapses made by TH-positive axons with either structures postsynaptic to VGluT-positive terminals or the terminals themselves. An apposition was defined as such, when two structures were apposed with no intervening structure. They are important to note because they could develop into symmetrical synapses in serial sections beyond the six examined. Furthermore, if dopaminergic axons can release dopamine at extrasynaptic sites, appositions may be indicative of sites of functional interaction between the apposed structures. Asymmetrical synapses (Gray's type 1) were identified by the presence of presynaptic vesicle accumulation, a thick postsynaptic density, a widened synaptic cleft and cleft material. Symmetrical synapses (Gray's type 2) formed by THpositive axons were identified by presynaptic vesicle accumulation, a darkened postsynaptic membrane and cleft material. This differs from the classical criteria for symmetrical synapses (Gray, 1959; Mori, 1966), which include the presence of a widened synaptic cleft, because $\mathrm{TH}-$ positive synapses are often very transient and have very small membrane specializations. Varicose and inter-varicose segments of TH-positive axons could not be differentiated within the six serial sections examined because the diameters of these segments almost completely overlap (Pickel et al., 1981).

It is possible that any relationships observed between dopaminergic axons and excitatory synapses are random in nature and not selective. As a control for this we determined the spatial relationship between randomly selected structures in the striatum and the dopaminergic nigrostriatal axons. One section from the series of six serial sections on the electron microscope grid was selected at random. As above, a field of view within the depth of penetration of the immunoreagents was randomly selected at low magnification (i.e., no bias toward VGluT-positive terminals), and the structure whose plasma membrane was closest to the center of the field at a magnification of $\times 54,100$ was identified. Digital images of this structure were then recorded in each of the six serial sections. A total of 60 randomly selected structures were analyzed. The proportion of these structures either apposed by, or in synaptic contact with, a TH-positive structure was recorded. To control for differences in the sizes of randomly selected structures and VGluT-positive terminals and their postsynaptic targets, we measured the perimeters of the 60 randomly selected structures and of 60 VGluT-positive terminals and 65 structures postsynaptic to them. The frequency of structures being apposed to (or in synaptic contact with) a TH-positive axon was then corrected for the mean perimeter and expressed as the percentage apposed per micron of membrane.

The proportion of VGluT1-positive and VGluT2-positive synapses within $1.0 \mu \mathrm{m}$ and $0.5 \mu \mathrm{m}$ of a TH-positive axon was measured in the set of serial micrographs used to examine the VGluT-positive synapses. Measurements were made from the center of the VGluT-positive synapses (supplemental Fig. 1, available at www.jneurosci.org as supplemental material) to the nearest TH-positive axon over 6 serial sections. Only those VGluT-positive synapses that were positioned $>0.99 \mu \mathrm{m}$ and $>0.49 \mu \mathrm{m}$ from the edge of the captured image in all serial sections were analyzed for the respective studies (within $1.0 \mu \mathrm{m} n=33$, within $0.5 \mu \mathrm{m}$ $n=78$ ). Similarly, measurements were made from VGluT-positive synapses to the nearest synapse formed by TH-positive axons within the 6 serial sections.

To determine whether or not the frequency of TH-positive axons/ synapses being within $1.0 \mu \mathrm{m}$ and $0.5 \mu \mathrm{m}$ of glutamatergic synapses was random in nature, we examined the proximity of TH-positive axons/ synapses to an arbitrary point in the tissue. The frames randomly selected with no bias toward VGluT-positive terminals were used for this analysis. We measured the distance from the point at the center of the frame to the nearest $\mathrm{TH}$-positive axon or TH-positive synapse within the serial sections and noted the proportion of these that were within $1.0 \mu \mathrm{m}$ and 0.5 $\mu \mathrm{m}$. Only random points that remained $>0.99 \mu \mathrm{m}$ and $>0.49 \mu \mathrm{m}$ from the edge the frame throughout the series were used for each respective study (within $1.0 \mu \mathrm{m}, n=50$; within $0.5 \mu \mathrm{m}, n=60$ ).
Data for the proportions of VGluT-positive synapses (and randomly selected points) within $0.5 \mu \mathrm{m}$ of $\mathrm{TH}$-positive axon or synapse were extrapolated from the volume examined, i.e., a cylinder of height 0.30 $\mu \mathrm{m}$ (six serial $50 \mathrm{~nm}$ sections) and radius $0.5 \mu \mathrm{m}$, to a sphere of radius 0.5 $\mu \mathrm{m}$ to give a better reflection of the distribution in three dimensional space $(n=138)$ (see Table 4$)$. Extrapolation of the frequency (f) of the spatial relationship between excitatory synapses and TH-positive structures from the cylinder of tissue examined $\left(f_{1}\right)$ to a sphere of $0.5 \mu \mathrm{m}$ diameter $\left(f_{2}\right)$ was performed by multiplying the ratio of the volume of the sphere/volume of the cylinder by the frequency as measured in the cylinder of tissue $\left(f_{1}\right)$ [ ( volume of sphere/volume of cylinder $\left.) \times f_{1}=f_{2}\right]$. In addition, the minimum radius of a sphere around VGluT1- and VGluT2positive synapses or randomly selected points necessary to reach a frequency $\left(\mathrm{f}_{2}\right)$ of $100 \%$, was calculated by assigning the value of $\mathrm{f}_{2}$ as $100 \%$, [(volume of sphere/volume of cylinder $) \times f_{1}=100 \%$ ], and solving the equation for the radius of the sphere. This reveals the minimum radius of a sphere around VGluT-positive synapses or randomly selected point which will include a TH-positive axon/synapse (i.e., the mean distance to a TH-positive axon/synapse).

\section{Results}

\section{Corticostriatal and thalamostriatal afferents contact different proportions of dendritic shafts and spines}

In agreement with previous studies (Lacey et al., 2005; Fujiyama et al., 2006; Raju et al., 2006, 2008), VGluT-positive terminals in the striatum made asymmetrical synapses (Gray's Type 1) mainly with dendritic spines and shafts. The 150 VGluT1-positive terminals that were examined formed a total of 170 synapses. The majority were with dendritic spines (95.9\%) (Fig. $1 A$ ), 3.5\% with dendritic shafts (Fig. $1 B$ ) and only one synapse with a cell body $(0.6 \%)$. The pattern was different for VGluT2-positive terminals (150 terminals, 173 synapses): $70.5 \%$ formed synapses with spines (Fig. 1C) and 29.5\% with dendritic shafts (Fig. 1D). In contrast to VGluT1-positive synapses, there was marked variability between animals in the proportions of synaptic targets of VGluT2-positive terminals (proportions of spines to shafts contacted were $61: 39 \%, 84: 16 \%$, and $67: 33 \%$ for the three animals). VGluT-positive terminals occasionally made synaptic contact with multiple structures (14.0\%) (Table 1, Fig. 2); most frequently they made contact with two spines (9.7\%) (Fig. 2C) but also with two shafts (1.3\%) (Fig. 2D), a shaft and a spine $(2.7 \%)$ (Fig. 2A) and on one occasion, three spines (0.3\%) (Fig. 2B).

\section{Glutamatergic and dopaminergic afferents converge on the same postsynaptic structures}

To define the spatial relationship between dopaminergic afferents and corticostriatal and thalamostriatal afferents, we next examined the proportion of structures postsynaptic to VGluT1and VGluT2-positive terminals that were apposed by, or in synaptic contact with, TH-positive axons (Table 2, Fig. 3). Any synaptic contacts made by $\mathrm{TH}$-positive axons with these structures is evidence for direct convergence of glutamatergic and dopaminergic signals in the striatum. Appositions by TH-positive axons show close relationships, which may indicate synaptic contacts beyond the scope of six serial sections examined or an indirect interaction by spill-over from dopaminergic synapses or possibly by the extrasynaptic release of dopamine. TH-positive terminals formed symmetrical synapses (Gray's Type 2) with both spines and dendritic shafts. Double-immunolabeling and serial-section analysis showed that $20.6 \%$ of the structures postsynaptic to VGluT1-positive terminals were apposed by TH-positive axons, of which $42.9 \%$ formed synapses (8.8\% of all structures postsynaptic to VGluT1-positive terminals) (Fig. $3 A, B$ ). Similarly, VGluT2 and TH double labeling revealed that $26.6 \%$ of the structures postsynaptic to the VGluT2-positive terminals were also 
apposed by TH-positive axons, of which $34.8 \%$ formed synapses $(9.2 \%$ of all structures postsynaptic to VGluT2-positive terminals; $\chi^{2}$ test, $p>0.05$ ) (Fig. $\left.3 C-F\right)$.

If only spines postsynaptic to VGluT1positive terminals are considered, $20.2 \%$ were apposed by a TH-positive axon. Of these, $42.4 \%$ were in synaptic contact which is equivalent to $8.6 \%$ of all spines postsynaptic to VGluT1-positive boutons. Six shafts were contacted by VGluT1positive terminals. Two of these were apposed by TH-positive axons, one of which was in synaptic contact.

When the spines postsynaptic to VGluT2-positive terminals are considered, $18.9 \%$ were apposed by TH-positive axons, of which $21.7 \%$ ( $4.1 \%$ of all spines postsynaptic to VGluT2-positive terminals) formed symmetrical synaptic contacts. Of the shafts postsynaptic to VGluT2-positive terminals, $45.1 \%$ were apposed by $\mathrm{TH}-$ positive axons and $47.8 \%$ of these $(21.6 \%$ of all dendritic shafts postsynaptic to VGluT2-positive terminals), received symmetrical synaptic contact from the $\mathrm{TH}$ positive axons.

In addition to apposing and forming synaptic contacts with structures postsynaptic to the VGluT-positive terminals, THpositive axons directly apposed the presynaptic VGluT-positive terminals to a similar degree. Of the VGluT1- and VGluT2positive terminals examined, $29.3 \%$ and $21.3 \%$, respectively, were apposed by a $\mathrm{TH}$ positive axon (Table 2, Fig. 4A). Although clear synaptic specializations were not observed, we occasionally observed membrane thickenings or a dense material on the apposing membranes. When apposing the VGluT-positive terminal the THpositive structure would often nestle at the edge of the synapse, apposing both the presynaptic and postsynaptic structure to a similar degree (Fig. 4B).

To determine whether the association between dopaminergic axons and VGluT-positive synapses was selective, we also measured the proportion of randomly selected structures that were apposed by, or in synaptic contact with dopaminergic axons. This analysis was performed in a similar way to the analysis described above; randomly selected structures (whose plasma membrane was closest to the center of the field), were examined in six serial sections. Of the 60 structures examined, $45.0 \%$ were apposed by TH-positive axons and $22.2 \%$ of these formed synapses $(10.0 \%$ of all randomly selected structures). However, randomly selected structures were larger than VGluT-positive terminals or their postsynaptic targets [the average perimeter of VGluT-positive terminals (mean $\pm \mathrm{SD}$ ) was $1.97 \pm 0.75 \mu \mathrm{m}, 1.59 \pm 0.80 \mu \mathrm{m}$ for their postsynaptic structures and $3.30 \pm 2.53 \mu \mathrm{m}$ for randomly selected structures]. When normalized for these differences, the frequency of VGluT-positive terminals apposed by TH-positive axons was 13\%, 15\% for their postsynaptic targets and 14\% for randomly selected structures. This indicates that there is unlikely
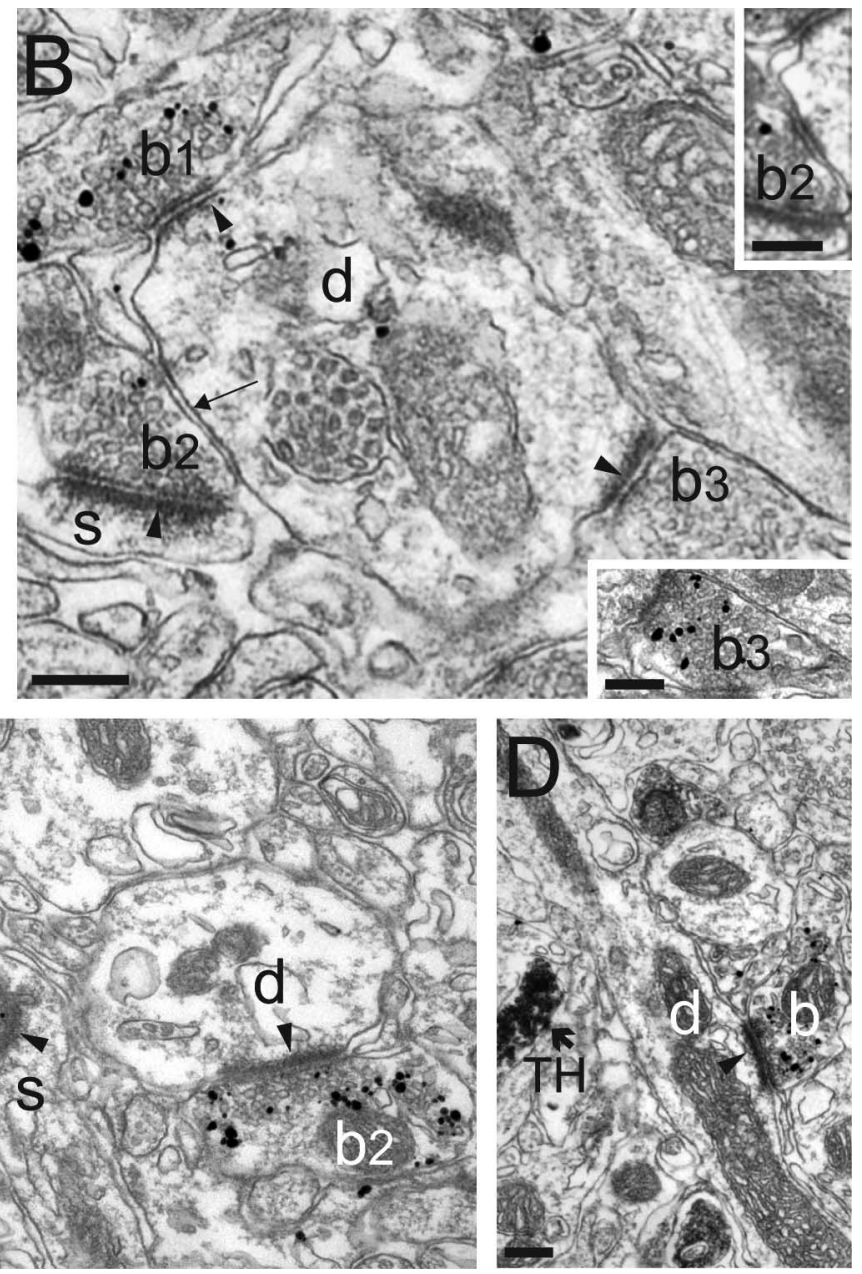

Figure 1. VGluT1- and VGluT2-positive terminals contact dendritic spines and shafts in the striatum. $\boldsymbol{A}$, VGluT1-positive shaft (d). A TH-positive axon (TH) is present in the field and is apposed to the spine in adjoining serial sections. $B$, VGluT1-positive (b1 and b3) make asymmetrical synapses (arrowheads) with a dendritic shaft (d). Another VGluT1-positive bouton (b2) (arrowheads) with a spine (s) and a dendritic shaft (d), respectively. D, A VGluT2-positive bouton (b) makes asymmetrical synaptic contact (arrowhead) with a dendritic shaft (d). A TH-positive axon (TH) is in the vicinity. Scale bars, $200 \mathrm{~nm}$.

Table 1. Single and multiple targets of individual VGluT1- and VGluT2-positive terminals

\begin{tabular}{lcc}
\hline Structure(s) contacted & VGluT1 terminals & VGluT2 terminals \\
\hline One spine & 126 & 90 \\
One shaft & 4 & 37 \\
One cell body & 1 & 0 \\
Two spines & 16 & 13 \\
Two shafts & 0 & 4 \\
One spine/one shaft & 2 & 6 \\
Three spines & 1 & 0 \\
\hline
\end{tabular}

to be selectivity in the location of, or targeting of dopaminergic axons within the striatum.

When the normalized apposition data for VGluT-positive terminals or their targets were categorized as VGluT1 or VGluT2positive and by their postsynaptic targets, similar values were obtained (Fig. 5). Fourteen percent of VGluT1-positive terminals 

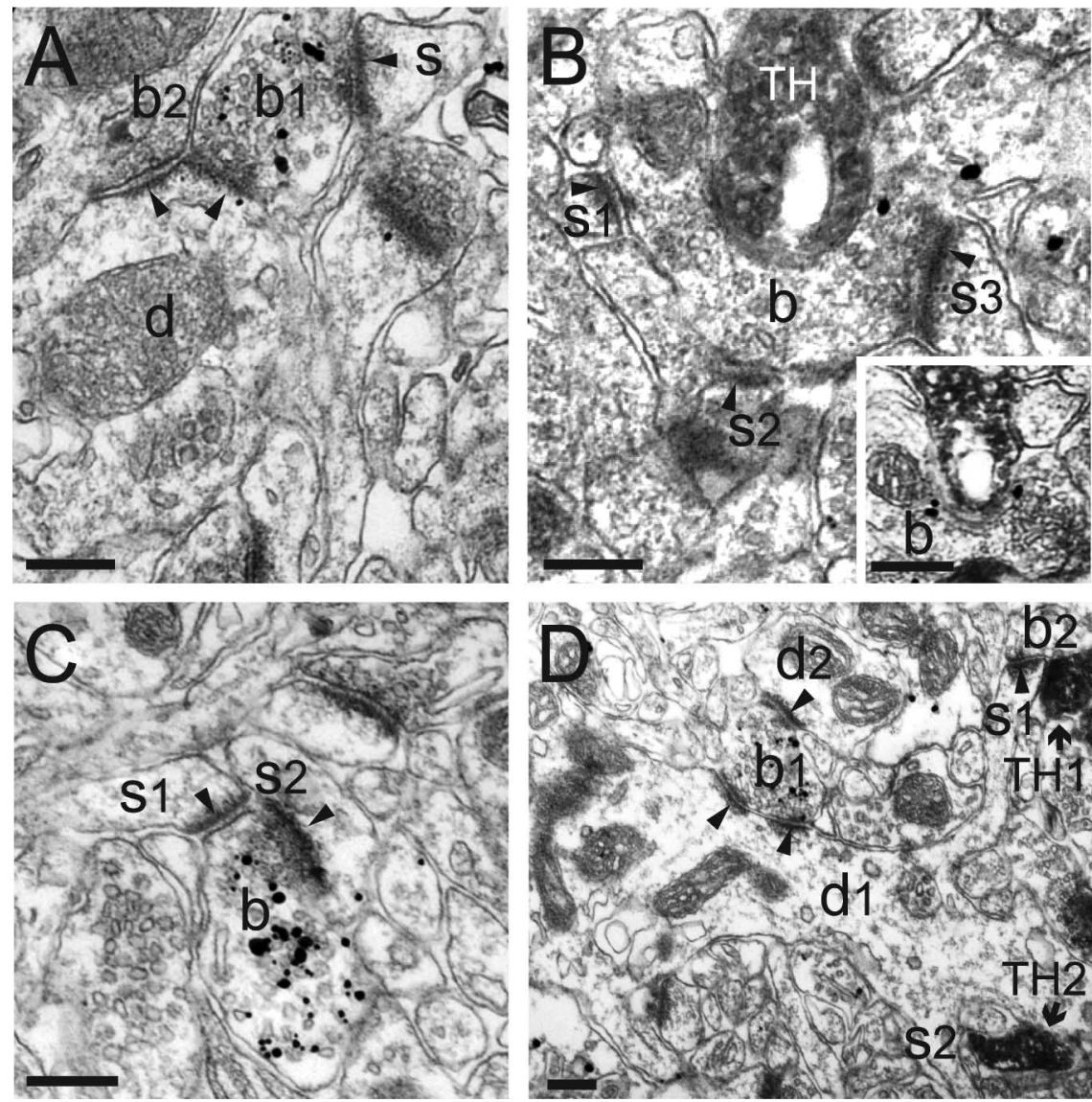

Figure 2. Individual VGluT1- and VGluT2-positive terminals often make multiple synaptic contacts. A, A VGluT1-positive bouton (b1) makes asymmetrical synaptic contacts (arrowheads) with a spine (s) and a dendritic shaft (d). The dendritic shaft (d) is also in asymmetrical synaptic contact with an unlabeled bouton (b2). B, A VGluT1-positive bouton (b) makes asymmetrical synaptic contact (arrowheads) with three spines ( $s 1$, s2 and s3; some contacts are seen more clearly in other serial sections of the six collected). Note that although there are only few immunogold particles overlying this bouton in this section, in the remaining five serial sections additional immunogold labeling was observed (inset) confirming that it was VGluT1-positive. Also note that this bouton is apposed by a TH-positive axon (TH). C, A VGluT2-positive bouton (b) makes asymmetrical synaptic contacts (arrowheads) with two spines (s1 and s2). D, A VGluT2-positive bouton (b1) makes asymmetrical synaptic contacts (arrowheads) with two dendritic shafts ( $\mathrm{d} 1$ and $\mathrm{d} 2$ ). Two spines ( $\mathrm{s} 1$ and $\mathrm{s} 2$ ) are seen arising from the dendrite, $\mathrm{d} 1$, and both are in close proximity to TH-positive axons (TH1 and TH2, respectively). One of these spines (s1) is in asymmetrical synaptic contact (arrowhead) with an unlabeled bouton (b2). Scale bars, $200 \mathrm{~nm}$.

Table 2. The numbers of VGluT1- and VGluT2-positive terminals, their postsynaptic targets, and random control structures apposed and in synaptic contact with THpositive axons

\begin{tabular}{lrll}
\hline & $\begin{array}{l}\text { Total } \\
\text { examined }\end{array}$ & $\begin{array}{l}\text { Number apposed by } \\
\text { TH-positive axons }\end{array}$ & $\begin{array}{l}\text { Number in synaptic contact } \\
\text { with TH-positive axons }\end{array}$ \\
\hline VGluT1 terminals & 150 & 44 & - \\
$\quad$ Postsynaptic spines & 163 & 33 & 14 \\
Postsynaptic dendrites & 6 & 2 & 1 \\
VGluT2 terminals & 150 & 32 & - \\
$\quad$ Postsynaptic spines & 122 & 23 & 5 \\
$\quad$ Postsynaptic dendrites & 51 & 23 & 11 \\
Random structures & 60 & 27 & 6 \\
\hline
\end{tabular}

-, No clear snyapses were observed.

contacting spines and $13 \%$ of their postsynaptic spines were apposed by a TH-positive axon. Eleven percent of VGluT2-positive terminals that contact spines were apposed by TH-positive axons as were $14 \%$ of these spines themselves. None of the VGluT1positive terminals contacting shafts were apposed by a THpositive axon and $14 \%$ of the shafts they contacted were apposed. However, very few VGluT1-positive terminals contacted shafts and for this reason a less robust comparison can be made. This was not the case for VGluT2-positive terminals. Fourteen percent of VGluT2-positive terminals contacting shafts were apposed by a TH-positive axon and $17 \%$ of these shafts were apposed themselves by a TH-positive axon.

When normalized for their perimeters, $5.7 \%$ of structures postsynaptic to VGluTpositive terminals formed synapses with TH-positive axons. The normalized incidence of randomly selected structures forming synapses with $\mathrm{TH}$-positive axons was $3.0 \%$ per micron of their perimeter.

\section{Excitatory synapses are in close proximity to dopaminergic axons} As shown above, we observed in serial sections that only $20-30 \%$ of structures postsynaptic to excitatory terminals are apposed by TH-positive axons and $\sim 9 \%$ are in synaptic contact. Although this may be an underestimate because only six serial sections were examined, it still leaves a large proportion of postsynaptic structures that are not apposed to, or in synaptic contact with, TH-positive axons. However, these may still be influenced by released dopamine. It has been proposed that synaptic spill-over of dopamine or extrasynaptically released dopamine may diffuse for up to $2 \mu \mathrm{m}$ in concentrations sufficient to stimulate D1 receptors and 7-8 $\mu \mathrm{m}$ in concentrations sufficient to stimulate D2 receptors (Rice and Cragg, 2008) (see also Gonon, 1997; Cragg and Rice, 2004).

We therefore quantified the proportion of VGluT-positive synapses that were within $0.5 \mu \mathrm{m}$ and $1.0 \mu \mathrm{m}$ of a $\mathrm{TH}$-positive axon or synapse (Table 3 ; supplemental Fig. 1, available at www.jneurosci.org as supplemental material). Of the VGluT1positive synapses, $48.5 \%$ were within $0.5 \mu \mathrm{m}$ and $90.9 \%$ were within $1.0 \mu \mathrm{m}$ of a TH-positive axon. Similarly, $46.7 \%$ of VGluT2-positive synapses were within $0.5 \mu \mathrm{m}$ and $72.7 \%$ were within $1.0 \mu \mathrm{m}$ ( $\chi^{2}$ test, $\left.p>0.05\right)$. When considering TH-positive synapses, $9.1 \%$ of VGluT1-positive synapses were within $0.5 \mu \mathrm{m}$ and $36.4 \%$ were within $1.0 \mu \mathrm{m}$. Similarly, $4.4 \%$ of VGluT2positive synapses were within $0.5 \mu \mathrm{m}$ of a TH-positive synapse and $9.1 \%$ and within $1.0 \mu \mathrm{m}$, figures that are similar to VGluT1positive synapses ( $\chi^{2}$ test, $p>0.05$ ). Pooling of the VGluT1 and VGluT2 data revealed that 47.4 and $84.8 \%$ of VGluT-positive synapses were within 0.5 and $1.0 \mu \mathrm{m}$ of a TH-positive axon, respectively. Furthermore, 6.4 and $27.3 \%$ of VGluT-positive synapses, respectively, were within 0.5 and $1.0 \mu \mathrm{m}$ of a TH-positive synapse.

As a control we determined frequency of randomly selected points (the center of frames randomly selected from the same tissue) being within 0.5 or $1.0 \mu \mathrm{m}$ of a $\mathrm{TH}$-positive axon or synapse, in a similar manner to that described above (Table 3; supplemental Fig. 1, available at www.jneurosci.org as supplemental material). In $43.3 \%$ of the cases the randomly selected points 
were within $0.5 \mu \mathrm{m}$ and $88.0 \%$ were within $1.0 \mu \mathrm{m}$ of a TH-positive axon respectively, values that are similar to the proportions for VGluT-positive synapses $\left(\chi^{2}\right.$ test, $p>$ $0.10)$. When considering TH-positive synapses, 5.0 and $18.0 \%$ of randomly selected points were within 0.5 and $1.0 \mu \mathrm{m}$, respectively, again similar to the values for VGluT-positive synapses $\left(\chi^{2}\right.$ test, $p>$ $0.15)$.

The distances between dopaminergic axons/synapses and VGluT1-positive synapses, VGluT2-positive synapses or randomly selected points (within $1 \mu \mathrm{m}$ ) were assessed for the uniformity of their distributions. Each set of measurements was seen to match the distribution of data of a normally distributed population (Kolmogorov-Smirnov test) except for the measurement between $\mathrm{TH}$-positive synapses and VGluT2-positive synapses because this was only observed in one case.

Our data are derived from the analysis of six serial $50 \mathrm{~nm}$ sections, so the frequency of occurrence of VGluT-positive synapses and randomly selected points within 0.5 or $1.0 \mu \mathrm{m}$ of a TH-positive axon or synapse is an underestimate of the true frequencies in three dimensions (i.e., we examined a cylinder of tissue with height of $0.3 \mu \mathrm{m}$ and radius 0.5 or $1.0 \mu \mathrm{m})$. Assuming uniformity of distribution of $\mathrm{TH}$ positive structures throughout the striatum (as shown by the KolmogorovSmirnov test), we can extrapolate the data we obtained from the cylinder of tissue to a sphere of tissue that will more closely reflect the frequencies of interactions in three dimensions.

The proportions of VGluT1-positive synapses, VGluT2-positive synapses, and randomly selected points that are located within $0.5 \mu \mathrm{m}$ of a TH-positive axon, extrapolated to a sphere of tissue, were 108 , 104 and 96\%, respectively (Table 4). This indicates that every VGluT-positive synapse and virtually every randomly selected point within the striatum will be within $0.5 \mu \mathrm{m}$ of a TH-positive axon. The radii of the spheres around VGluTpositive synapses or randomly selected points that will include a $\mathrm{TH}$-positive axon on every occasion (i.e., a proportion of $100 \%$ ), are $0.49,0.49$ and $0.51 \mu \mathrm{m}$ for VGluT1-postive synapses, VGluT2positive synapses and random points, respectively (Table 4 ).

If we consider $\mathrm{TH}$-positive synapses, rather than $\mathrm{TH}$-positive axons, extrapolated to a sphere, then 20 , 10 and $11 \%$ of VGluT1-positive synapses, VGluT2-positive synapses, and randomly selected points, respectively, will be within $0.5 \mu \mathrm{m}$ of a TH-positive synapse (Table 4). Similar to above, the radii of the spheres around VGluT1-positive synapses, VGluT2-
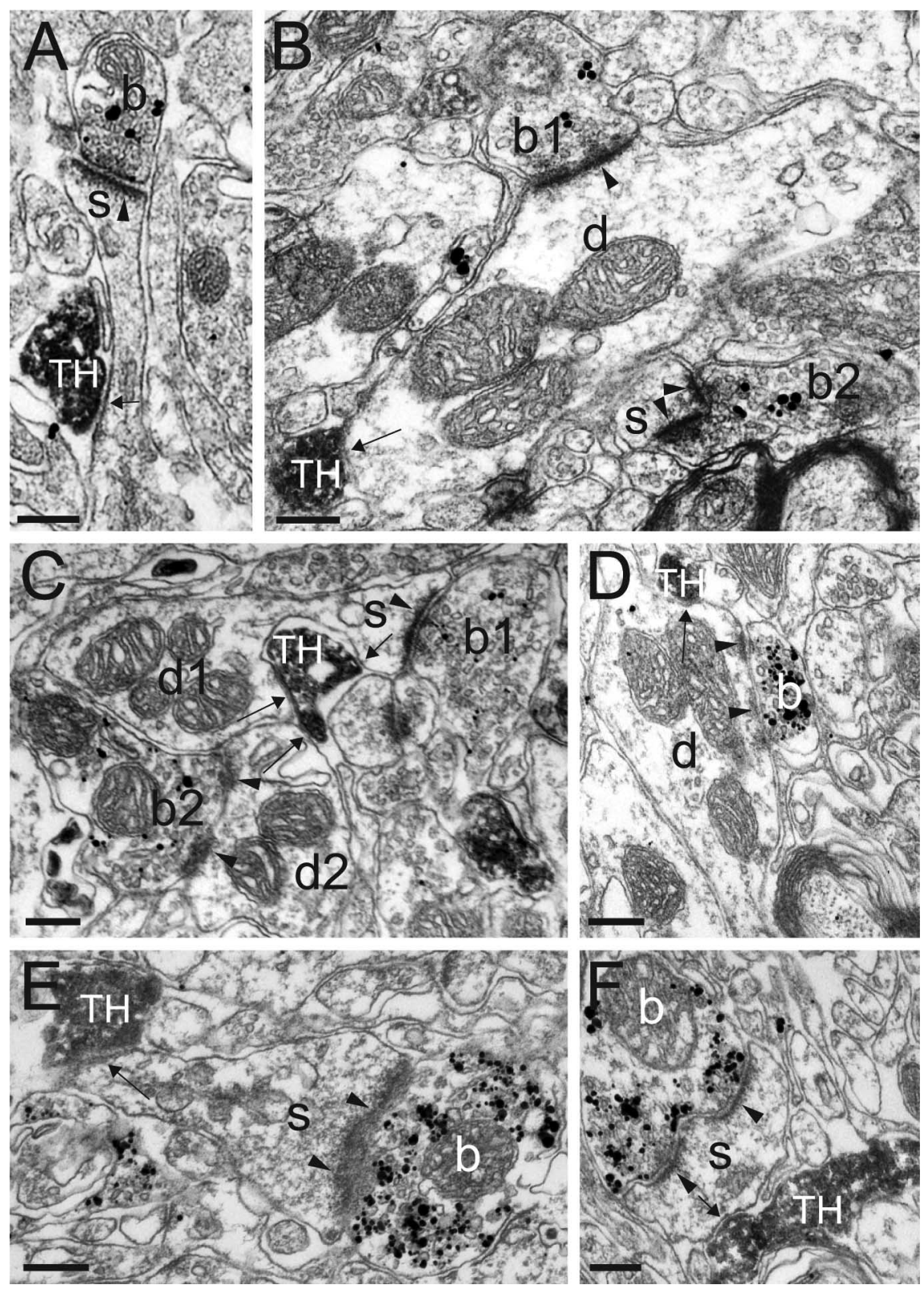

Figure 3. Striatal spines and dendritic shafts postsynaptic to VGlut1- or VGluT2-positive terminals are apposed by, or in synaptic contact with, TH-positive terminals. A, A VGluT1-positive bouton (b) makes asymmetrical synaptic contact (arrowhead) with the head of long thin spine (s). A TH-positive terminal (TH) makes symmetrical synaptic contact (arrow) with the neck of the same spine. B, A VGluT1-positive bouton (b1) makes asymmetrical synaptic contact with a dendritic shaft (d) which is apposed (arrow) by a TH-positive axon (TH). Within the vicinity, another VGluT1-positive bouton (b2) makes asymmetrical synaptic contact (arrowheads) with a spine (s). C, A VGluT2-positive bouton (b1) makes asymmetrical synaptic contact (arrowhead) with a spine (s) that arises from a dendritic shaft (d1). A TH-positive bouton (TH) makes symmetrical synaptic contact (arrows) with both the spine (s) and the dendritic shaft (d1) as well as neighboring dendritic shaft (d2). The latter also receives asymmetrical synaptic input (arrowheads) from a VGluT2-positive bouton (b2). D, VGluT2-positive bouton (b) makes asymmetrical synaptic contact (arrowhead) with a dendritic shaft (d) which is in symmetrical synaptic contact (arrow) with TH-positive terminal (TH). $\boldsymbol{E}$, A VGluT2-positive bouton (b) makes asymmetrical synaptic contact (arrowheads) with a spine (s) which is synaptically contacted at its neck (symmetrical synapse: arrow) by a TH-positive terminal (TH). $\boldsymbol{F}$, A VGluT2-positive bouton (b) makes asymmetrical synaptic contact (arrowheads) with a spine(s). ATH-positive terminal (TH) forms a symmetrical synapse (arrow) onto the opposite side of the same spine. The symmetrical synapses in this figure were identified by three criteria: a presynaptic accumulation of synaptic vesicles, synaptic cleft material and a darkened postsynaptic membrane (see Materials and Methods). Scale bars, $200 \mathrm{~nm}$. positive synapses or randomly selected points that will include a TH-positive axon on every occasion (i.e., a proportion of 100\%), are $0.85,1.08$ and $1.04 \mu \mathrm{m}$ for VGluT1-positive synapses, VGluT2-positive synapses and randomly selected points respectively (Table 4). It should be remembered that these figures are 

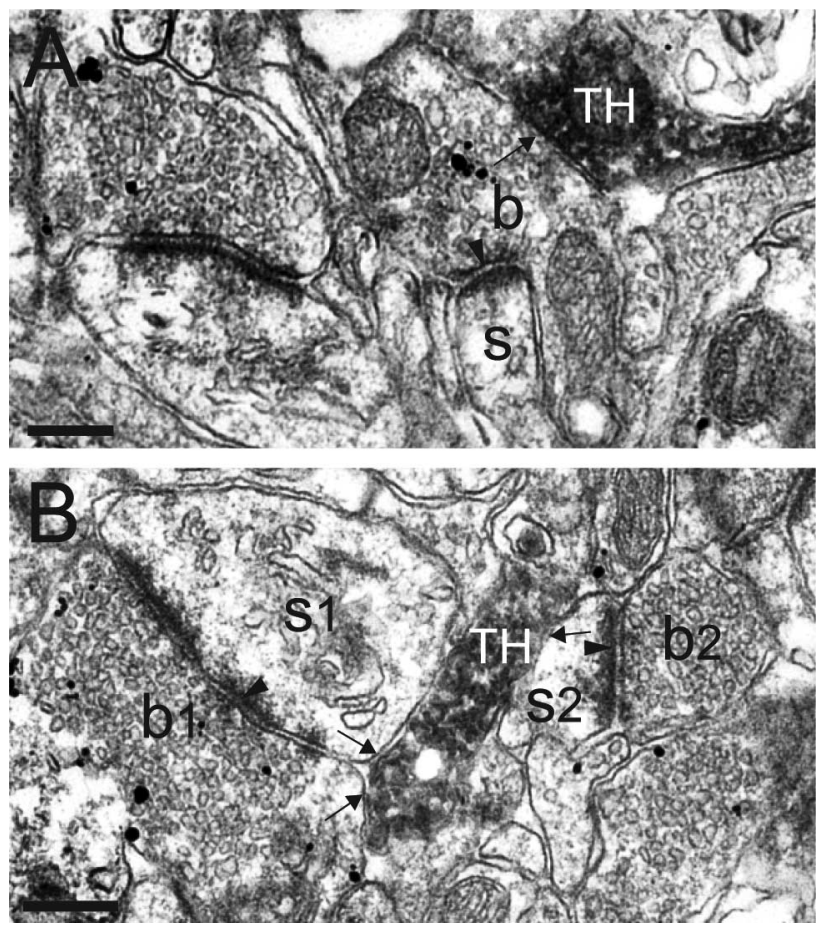

Figure 4. TH-positive axons often appose VGluT1- and VGluT2-positive terminals. A, A THpositive terminal (TH) is closely apposed (arrow) to a VGluT1-positive bouton (b), which in turn makes asymmetrical synaptic contact (arrowhead) with a spine (s). B, A TH-positive axon (TH) nestles at the edge of an asymmetrical synapse (arrowhead) between a VGluT1-positive bouton (b1) and a spine (s1). It is closely apposed (arrows) to b1, s1 and an additional spine, s2, which makes asymmetrical contact with an unlabeled bouton (b2). Scale bars, $200 \mathrm{~nm}$.

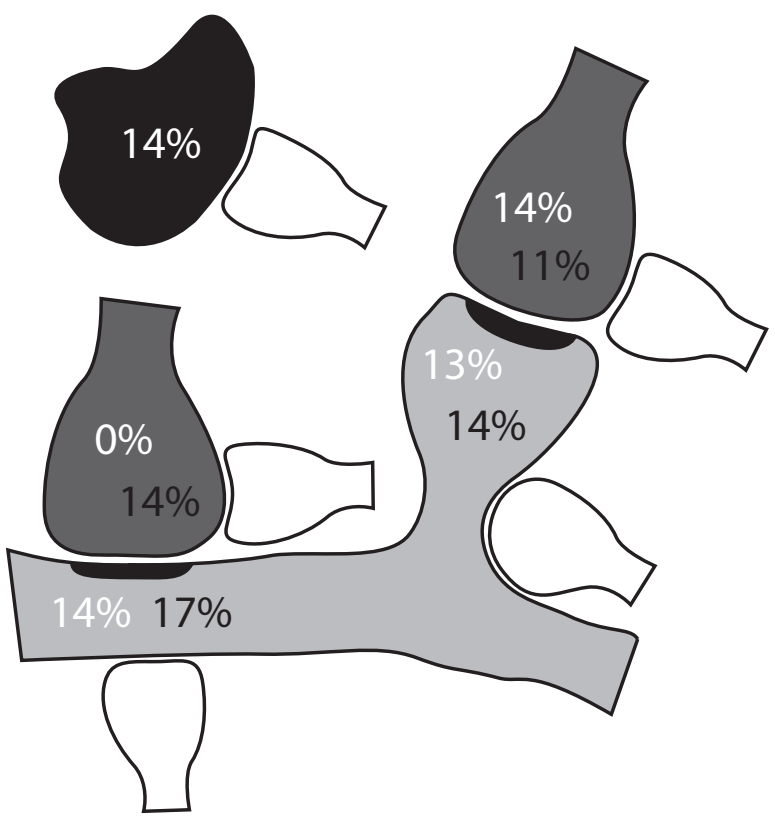

Figure 5. Schematic showing the proportions of VGluT1-positive (white numbers) or VGluT2-positive (black numbers) terminals (dark gray), their postsynaptic spines/shafts (light gray) and random control structures (black) that were apposed by TH-positive axons (white). Figures normalized for length of perimeter.

best estimates from the available data, which is in some cases comes from a relatively small sample size but they show that all structures within the striatum are within $\sim 1 \mu \mathrm{m}$ of a TH-positive synapse.

\section{Discussion}

The interaction between glutamatergic corticostriatal afferents and dopaminergic nigrostriatal afferents is a critical factor in the expression of basal ganglia function. The aims of the present study were to compare the spatial relationships between dopaminergic nigrostriatal axons and synapses and both of the principal glutamatergic afferents of the striatum originating in the cortex and the thalamus, and to determine whether any relationship identified is a selective association or random in nature. The main findings relating to these issues are twofold. First, that the spines and dendrites postsynaptic to thalamostriatal terminals are apposed and innervated by dopaminergic axons to a similar degree as structures postsynaptic to corticostriatal terminals. It is thus likely that an additional critical factor in the expression of basal ganglia function is the interaction between glutamatergic thalamostriatal afferents and dopaminergic nigrostriatal afferents. Our second principal finding is that the dopaminergic innervation of the striatum conforms to a dense three-dimensional lattice-work in which the probability of being apposed by, or in synaptic contact with a dopaminergic axon, is dependent on size. The implications of this are far-reaching and suggest that the ultimate distribution of dopaminergic axons in the striatum is unlikely to be a targeted phenomenon.

\section{The relationship between glutamatergic synapses and dopaminergic axons in the striatum}

Our use of serial-section analysis and the fact that we were able to analyze separately corticostriatal and thalamostriatal synapses by the use of antibodies to different vesicular glutamate transporters (Fremeau et al., 2001), enabled us to provide detailed quantitative data of the relationships between identified excitatory terminals and dopaminergic axons within the striatum. In addition to dopamine being released at synapses, it has been proposed that it may also be released at extrasynaptic sites and by spill-over from synapses. However, it is not yet possible to unequivocally identify nonsynaptic release sites. We therefore quantified the relationship between excitatory synapses and dopaminergic axons at the two extremes: the number of dopaminergic axons that were directly apposed to the membrane of structures in the striatum and the number of dopaminergic axons making synaptic connections. The former represents the maximum possible number of sites of interaction with excitatory synapses (if we assume that dopamine release can occur at all parts of an axon), whereas the latter represents the most conservative estimate of sites of interaction. With this approach we identified that $\sim 20 \%$ of spines postsynaptic to corticostriatal terminals were apposed by a dopaminergic axon and, in about half of these cases (8.8\%), the dopaminergic axon formed a synapse. These findings support the widely held view of the relationship between dopaminergic axons and corticostriatal synapses and data from anterograde tracing studies combined with TH immunocytochemistry in rats and monkeys (Bouyer et al., 1984; Smith et al., 1994). We similarly found that $\sim 19 \%$ of spines postsynaptic to thalamostriatal terminals were apposed by a dopaminergic axon, approximately one fifth of which also formed a synapse. Furthermore, $45 \%$ of dendritic shafts postsynaptic to thalamostriatal terminals were apposed by dopaminergic axons and approximately half were synaptic. The differences between spines and dendrites are presumably because of the larger size of dendrites. However, on average, the proportions were similar for corticostriatal and thalamostriatal synapses.

Our data thus support the notion that one of the principal sites of interaction of dopamine and glutamate in the striatum is 
Table 3. Numbers and percentages of VGluT1- and VGluT2-positive synapses or random control points from the same tissue that were within $0.5 \mu \mathrm{m}$ and $1.0 \mu \mathrm{m}$ of a THpositive axon or synapse in six serial sections

\begin{tabular}{|c|c|c|c|c|c|}
\hline & \multirow[b]{2}{*}{ Total examined } & \multicolumn{2}{|c|}{ Within $0.5 \mu \mathrm{m}$ of a TH-positive axon } & \multicolumn{2}{|c|}{ Within $0.5 \mu \mathrm{m}$ of a TH-positive synapse } \\
\hline & & Number & Percentage & Number & Percentage \\
\hline VGluT1 synapses & 33 & 16 & 48.5 & 3 & 9.1 \\
\hline VGluT2 synapses & 45 & 21 & 46.7 & 2 & 4.4 \\
\hline VGluT synapses & 78 & 37 & 47.4 & 5 & 6.4 \\
\hline \multirow[t]{3}{*}{ Random points } & 60 & 26 & 43.3 & 3 & 5.0 \\
\hline & & \multicolumn{2}{|c|}{ Within $1.0 \mu \mathrm{m}$ of a TH-positive axon } & \multicolumn{2}{|c|}{ Within $1.0 \mu \mathrm{m}$ of a TH-positive synapse } \\
\hline & Total examined & Number & Percentage & Number & Percentage \\
\hline VGluT1 synapses & 22 & 20 & 90.9 & 8 & 36.4 \\
\hline VGluT2 synapses & 11 & 8 & 72.7 & 1 & 9.1 \\
\hline VGluT synapses & 33 & 28 & 84.8 & 9 & 27.3 \\
\hline Random points & 50 & 44 & 88.0 & 9 & 18.0 \\
\hline
\end{tabular}

Table 4. Experimental data obtained from the cylinder of tissue in six serial sections extrapolated to the volume of a sphere

\begin{tabular}{lllll}
\hline & Total & $\begin{array}{l}\text { TH-positive axon }\left(\mathrm{f}_{1}\right) \\
\text { (experimental data) }\end{array}$ & $\begin{array}{l}\text { TH-positive axon }\left(\mathrm{f}_{2}\right) \\
\text { (extrapolated from data) }\end{array}$ & $\begin{array}{l}\text { Radius }(\mathrm{r}) \text { of sphere } \\
\text { (extrapolated from data) }\end{array}$ \\
\hline VGluT1 synapses & 33 & 48.5 & 107.7 & 0.49 \\
VGluT2 synapses & 45 & 46.7 & 103.7 & 0.49 \\
VGluT synapses & 78 & 47.4 & 105.4 & 0.49 \\
Random points & 60 & 43.3 & 96.3 & 0.51 \\
\hline & & TH-positive synapse $\left(\mathrm{f}_{1}\right)$ & TH-positive synapse $\left(\mathrm{f}_{2}\right)$ & Radius $(\mathrm{r})$ of sphere \\
& Total & (experimental data) & (extrapolated from data) & (extrapolated from data) \\
\hline VGluT1 synapses & 33 & 9.1 & 20.2 & 0.85 \\
VGluT2 synapses & 45 & 4.4 & 9.9 & 1.08 \\
VGluT synapses & 78 & 6.4 & 14.2 & 0.96 \\
Random points & 60 & 5.0 & 11.1 & 1.04 \\
\hline
\end{tabular}

Proportions of VGluT1- and VGluT2-positive synapses or random points within $0.5 \mu \mathrm{m}$ of a TH-positive axon or synapse in serial sections $\left(\mathrm{f}_{1}\right)$ or a sphere $\left(\mathrm{f}_{2}\right)$ are shown together with the minimum radius $(r)$ of a sphere around each that is necessary to include a TH-positive axon/synapse (100\%).

at postsynaptic sites, i.e., mainly dendritic spines (Bouyer et al., 1984; Freund et al., 1984; Smith and Bolam, 1990; Smith et al., 1994). We also demonstrate that it is both corticostriatal and thalamostriatal afferents that are in a position to be influenced by the dopaminergic afferents (see also Pinto et al., 2003). We propose therefore that the modulatory or gating effect of dopamine on glutamate transmission applies equally to corticostriatal and thalamostriatal synapses. Furthermore, long and short term plasticity of thalamostriatal synapses (Smeal et al., 2007; Ding et al., 2008; Raju et al., 2008), like that of the corticostriatal synapses, is likely, at least in part, to be dependent on released dopamine. These findings, together with those of previous studies (Dubé et al., 1988; Xu et al., 1991; Lapper and Bolam, 1992; Lacey et al., 2005, 2007; McHaffie et al., 2005; Raju et al., 2006), suggest that the thalamostriatal projections exhibit a rich and diverse complexity of function on a par with that of the corticostriatal projections.

\section{The lattice of dopaminergic axons in the striatum}

If indeed dopaminergic modulation of glutamate transmission is so central to basal ganglia function (Surmeier et al., 2007), the question arises as to why such a seemingly small proportion of glutamatergic synapses form convergent contacts with dopaminergic terminals. Are the glutamatergic synapses that do not have a dopaminergic terminal in contact with their postsynaptic structure in a position to be equally affected by released dopamine? In addition to synaptic transmission, it has been proposed that dopaminergic transmission may also occur by 'volume transmission' as a consequence of spill-over of synaptically released dopamine or release at nonsynaptic sites (Agnati et al., 1995). The "sphere-of-influence" of released dopamine is likely to depend on many factors including quantal size and the density and distribution of dopamine transporters and receptors. However, it has been proposed that the sphere-of-influence of dopamine spill-over in a concentration sufficient to stimulate dopamine receptors, has a radius of $2-8 \mu \mathrm{m}$ (Rice and Cragg, 2008) (see also Gonon, 1997; Cragg and Rice, 2004; Arbuthnott and Wickens, 2007). Our quantitative analysis revealed that a large proportion of VGluT-positive synaptic terminals themselves were directly apposed by TH-positive axons (29.3 and $21.3 \%$ for VGluTs 1 and 2 , respectively), and that $\sim 47$ and $85 \%$ of VGluT-positive synapses are within a radius of 0.5 and $1 \mu \mathrm{m}$ of a TH-positive axon, respectively, as recorded in serial sections. More importantly, 6 and $27 \%$ of VGluT-positive synapses are within 0.5 and $1 \mu \mathrm{m}$, respectively, of a $\mathrm{TH}$-positive synapse in serial sections. When our data are extrapolated to a sphere of radius $0.5 \mu \mathrm{m}$ (assuming the uniform distribution as demonstrated by the KolmogorovSmirnov test), more accurately reflecting the distribution in three dimensional space, all VGluT1- and VGluT2-positive synapses are within $0.5 \mu \mathrm{m}$ of a TH-positive axon and 20 and $10 \%$, respectively, within $0.5 \mu \mathrm{m}$ of a TH-positive synapse. If we look at this in a different way and ask what is the minimum radius of a sphere around a glutamatergic synapse that will always include a THpositive synapse (i.e., the mean distance to a TH-positive synapse in three dimensional space), we calculate it to be 0.85 and 1.08 $\mu \mathrm{m}$ for VGluT1- and VGluT2-positive synapses respectively. In view of the figures proposed by Rice and Cragg (2008) for the diffusion of dopamine from a synapse, our findings suggest that every glutamatergic synapse will be within overlapping spheresof-influence of synaptically released dopamine. Thus, all gluta- 
matergic synapses are likely to be within reach of a concentration of dopamine high enough to stimulate both high and low affinity receptors (Rice and Cragg, 2008).

On the face of it, our findings, and the consensus view in the literature, imply that the relationship between excitatory synapses and dopaminergic axons/synapses is a selective or targeted association. To test this directly, we examined the spatial relationship between randomly selected structures in the striatum and dopaminergic axons. We found that $\sim 43$ and $\sim 88 \%$ of the randomly selected structures are within $0.5 \mu \mathrm{m}$ and $1.0 \mu \mathrm{m}$ of a dopaminergic axon, respectively, in serial sections, figures that are remarkably close to those obtained in the analysis of glutamatergic (VGluT-positive) synapses (see above). Furthermore, when normalized for the length of plasma membrane that appears in the micrographs, the probability of a randomly selected structure being directly apposed by a dopaminergic axon was very similar to that of glutamatergic terminals and their postsynaptic targets $(14,13$, and $15 \%$ per micrometer plasma membrane, respectively). Similarly, the frequency of synapses formed by the dopaminergic axons on the randomly selected structures was very similar to the frequency of synapses formed on structures postsynaptic to glutamatergic axons.

Extrapolation of these data to a sphere of tissue to give a better indication the distribution in three dimensional space reveals that $96 \%$ of randomly selected points within the striatum are within $0.5 \mu \mathrm{m}$ of a TH-positive axon. The distance from randomly selected points to TH-positive synapses is larger $(1.04 \mu \mathrm{m})$ but indicates that all points in the striatum are within range of a "spill-over" concentration of synaptically released dopamine sufficient to stimulate receptors.

Our data support previous findings and suggestions relating to the organization of dopaminergic synapses in the striatum and quantitative aspects of striatal organization (Wilson, 1986; Groves et al., 1994; Ingham et al., 1998; Cragg and Rice, 2004; Arbuthnott and Wickens, 2007; Wilson, 2007; Rice and Cragg, 2008) and imply that there is no selectivity in the association of dopaminergic axons and glutamatergic synapses. Rather, they suggest that the organization of the dopaminergic nigrostriatal system is such that striatal neuropil is located within a dense, evenly spaced lattice-work of dopaminergic axons, and the probability of being apposed by a dopaminergic axon is principally dependent on the size of the structure. Thus, all similarly sized striatal structures have an approximately equal probability of being apposed by a dopaminergic axon. The organization is such that apposition by, and proximity to, a dopaminergic axon is random in nature.

\section{Conclusions}

We demonstrate that, like corticostriatal glutamatergic axon terminals, thalamostriatal terminals are in a position to be equally influenced by dopaminergic nigrostriatal axons. However, this does not represent a selective relationship, but rather it is simply a reflection of the density and organization of the nigrostriatal pathway. The specificity of the dopaminergic nigrostriatal system is most likely to lie in other factors like the timing of dopamine release and the distribution of dopamine receptors and transporters.

\section{References}

Agnati LF, Zoli M, Strömberg I, Fuxe K (1995) Intercellular communication in the brain: wiring versus volume transmission. Neuroscience 69:711-726.

Arbuthnott GW, Wickens J (2007) Space, time and dopamine. Trends Neurosci 30:62-69.
Björklund A, Lindvall O (1984) Dopamine-containing systems in the CNS. In: Handbook of chemical neuroanatomy, Vol. 2: Classical transmitters in the CNS Part 1 (Björklund A, Hökfelt T, eds), pp 55-122. Amsterdam: Elsevier.

Bolam JP, Hanley JJ, Booth PA, Bevan MD (2000) Synaptic organisation of the basal ganglia. J Anat 196:527-542.

Bouyer JJ, Park DH, Joh TH, Pickel VM (1984) Chemical and structural analysis of the relation between cortical inputs and tyrosine hydroxylasecontaining terminals in rat neostriatum. Brain Res 302:267-275.

Carlsson A (1959) The occurrence, distribution and physiological role of catecholamines in the nervous system. Pharmacol Rev 11:490-493.

Cragg SJ, Rice ME (2004) DAncing past the DAT at a DA synapse. Trends Neurosci 27:270-277.

DeLong MR (1990) Primate models of movement disorders of basal ganglia origin. Trends Neurosci 13:281-285.

Ding J, Peterson JD, Surmeier DJ (2008) Corticostriatal and thalamostriatal synapses have distinctive properties. J Neurosci 28:6483-6492.

Dubé L, Smith AD, Bolam JP (1988) Identification of synaptic terminals of thalamic or cortical origin in contact with distinct medium-size spiny neurons in the rat neostriatum. J Comp Neurol 267:455-471.

Fremeau RT Jr, Troyer MD, Pahner I, Nygaard GO, Tran CH, Reimer RJ, Bellocchio EE, Fortin D, Storm-Mathisen J, Edwards RH (2001) The expression of vesicular glutamate transporters defines two classes of excitatory synapse. Neuron 31:247-260.

Freund TF, Powell JF, Smith AD (1984) Tyrosine hydroxylaseimmunoreactive boutons in synaptic contact with identified striatonigral neurons, with particular reference to dendritic spines. Neuroscience 13:1189-1215.

Frotscher M, Rinne U, Hassler R, Wagner A (1981) Termination of cortical afferents on identified neurons in the caudate nucleus of the cat. A combined Golgi-EM degeneration study. Exp Brain Res 41:329-337.

Fujiyama F, Kuramoto E, Okamoto K, Hioki H, Furuta T, Zhou L, Nomura S, Kaneko T (2004) Presynaptic localization of an AMPA-type glutamate receptor in corticostriatal and thalamostriatal axon terminals. Eur J Neurosci 20:3322-3330.

Fujiyama F, Unzai T, Nakamura K, Nomura S, Kaneko T (2006) Difference in organization of corticostriatal and thalamostriatal synapses between patch and matrix compartments of rat neostriatum. Eur J Neurosci 24:2813-2824.

Gerfen CR, Wilson CJ (1996) The basal ganglia. In: Handbook of chemical neuroanatomy, Vol 12: Integrated systems of the CNS, Pt 3 (Swanson LW, Björklund A, Hökfelt T, eds), pp 371-468. Amsterdam: Elsevier.

Gonon F (1997) Prolonged and extrasynaptic excitatory action of dopamine mediated by $\mathrm{D} 1$ receptors in the rat striatum in vivo. J Neurosci 17:5972-5978.

Gray EG (1959) Axo-somatic and axo-dendritic synapses of the cerebral cortex: an electron microscope study. J Anat 93:420-433.

Graybiel AM (1995) Building action repertoires: memory and learning functions of the basal ganglia. Curr Opin Neurobiol 5:733-741.

Graybiel AM (2000) The basal ganglia. Curr Biol 10:R509-511.

Groenewegen HJ, Berendse HW (1994) The specificity of the 'nonspecific' midline and intralaminar thalamic nuclei. Trends Neurosci 17:52-57.

Groves PM, Linder JC, Young SJ (1994) 5-Hydroxydopamine-labeled dopaminergic axons: three-dimensional reconstructions of axons, synapses and postsynaptic targets in rat neostriatum. Neuroscience 58:593-604.

Härtig W, Riedel A, Grosche J, Edwards RH, Fremeau RT Jr, Harkany T, Brauer K, Arendt T (2003) Complementary distribution of vesicular glutamate transporters 1 and 2 in the nucleus accumbens of rat: relationship to calretinin-containing extrinsic innervation and calbindinimmunoreactive neurons. J Comp Neurol 465:1-10.

Herzog E, Bellenchi GC, Gras C, Bernard V, Ravassard P, Bedet C, Gasnier B, Giros B, El Mestikawy S (2001) The existence of a second vesicular glutamate transporter specifies subpopulations of glutamatergic neurons. J Neurosci 21:RC181.

Ingham CA, Hood SH, Taggart P, Arbuthnott GW (1998) Plasticity of synapses in the rat neostriatum after unilateral lesion of the nigrostriatal dopaminergic pathway. J Neurosci 18:4732-4743.

Kaneko T, Fujiyama F (2002) Complementary distribution of vesicular glutamate transporters in the central nervous system. Neurosci Res 42:243-250.

Kemp JM, Powell TP (1971) The synaptic organization of the caudate nucleus. Philos Trans R Soc Lond B Biol Sci 262:403-412. 
Kerr JN, Wickens JR (2001) Dopamine D-1/D-5 receptor activation is required for long-term potentiation in the rat neostriatum in vitro. J Neurophysiol 85:117-124.

Lacey CJ, Boyes J, Gerlach O, Chen L, Magill PJ, Bolam JP (2005) GABA(B) receptors at glutamatergic synapses in the rat striatum. Neuroscience 136:1083-1095.

Lacey CJ, Bolam JP, Magill PJ (2007) Novel and distinct operational principles of intralaminar thalamic neurons and their striatal projections. J Neurosci 27:4374-4384.

Lapper SR, Bolam JP (1992) Input from the frontal cortex and the parafascicular nucleus to cholinergic interneurons in the dorsal striatum of the rat. Neuroscience 51:533-545.

Magill PJ, Bolam JP, Bevan MD (2001) Dopamine regulates the impact of the cerebral cortex on the subthalamic nucleus-globus pallidus network. Neuroscience 106:313-330.

McHaffie JG, Stanford TR, Stein BE, Coizet V, Redgrave P (2005) Subcortical loops through the basal ganglia. Trends Neurosci 28:401-407.

Moore RY, Card JP (1984) Noradrenaline-containing neuron systems. In: Handbook of chemical neuroanatomy, Vol 2: Classical transmitters in the CNS Pt 1 (Björklund A, Hökfelt T, eds), pp 123-156. Amsterdam: Elsevier.

Mori S (1966) Some observations on the fine structure of the corpus striatum of the rat brain. Z Zellforsch Mikrosk Anat 70:461-488.

Pickel VM, Beckley SC, Joh TH, Reis DJ (1981) Ultrastructural immunocytochemical localization of tyrosine hydroxylase in the neostriatum. Brain Res 225:373-385.

Pinto A, Jankowski M, Sesack SR (2003) Projections from the paraventricular nucleus of the thalamus to the rat prefrontal cortex and nucleus accumbens shell: ultrastructural characteristics and spatial relationships with dopamine afferents. J Comp Neurol 459:142-155.

Prasad BM, Amara SG (2001) The dopamine transporter in mesencephalic cultures is refractory to physiological changes in membrane voltage. J Neurosci 21:7561-7567.

Raju DV, Shah DJ, Wright TM, Hall RA, Smith Y (2006) Differential synaptology of vGluT2-containing thalamostriatal afferents between the patch and matrix compartments in rats. J Comp Neurol 499:231-243.

Raju DV, Ahern TH, Shah DJ, Wright TM, Standaert DG, Hall RA, Smith Y (2008) Differential synaptic plasticity of the corticostriatal and thalamostriatal systems in an MPTP-treated monkey model of parkinsonism. Eur J Neurosci 27:1647-1658.

Reynolds JN, Hyland BI, Wickens JR (2001) A cellular mechanism of reward-related learning. Nature 413:67-70.
Rice ME, Cragg SJ (2008) Dopamine spillover after quantal release: rethinking dopamine transmission in the nigrostriatal pathway. Brain Res Rev 58:303-313.

Shen W, Flajolet M, Greengard P, Surmeier DJ (2008) Dichotomous dopaminergic control of striatal synaptic plasticity. Science 321:848-851.

Smeal RM, Gaspar RC, Keefe KA, Wilcox KS (2007) A rat brain slice preparation for characterizing both thalamostriatal and corticostriatal afferents. J Neurosci Methods 159:224-235.

Smith AD, Bolam JP (1990) The neural network of the basal ganglia as revealed by the study of synaptic connections of identified neurones. Trends Neurosci 13:259-265.

Smith Y, Bennett BD, Bolam JP, Parent A, Sadikot AF (1994) Synaptic relationships between dopaminergic afferents and cortical or thalamic input in the sensorimotor territory of the striatum in monkey. J Comp Neurol 344:1-19.

Smith Y, Bevan MD, Shink E, Bolam JP (1998) Microcircuitry of the direct and indirect pathways of the basal ganglia. Neuroscience 86:353-387.

Smith Y, Raju DV, Pare JF, Sidibé M (2004) The thalamostriatal system: a highly specific network of the basal ganglia circuitry. Trends Neurosci 27:520-527.

Somogyi P, Bolam JP, Smith AD (1981) Monosynaptic cortical input and local axon collaterals of identified striatonigral neurons. A light and electron microscopic study using the Golgi-peroxidase transportdegeneration procedure. J Comp Neurol 195:567-584.

Surmeier DJ, Ding J, Day M, Wang Z, Shen W (2007) D1 and D2 dopaminereceptor modulation of striatal glutamatergic signaling in striatal medium spiny neurons. Trends Neurosci 30:228-235.

Villalba RM, Raju DV, Hall RA, Smith Y (2006) GABA(B) receptors in the centromedian/parafascicular thalamic nuclear complex: an ultrastructural analysis of $\mathrm{GABA}(\mathrm{B}) \mathrm{R} 1$ and $\mathrm{GABA}(\mathrm{B}) \mathrm{R} 2$ in the monkey thalamus. J Comp Neurol 496:269-287.

Wilson CJ (1986) Three-dimensional analysis of dendritic spines by means of HVEM. J Electron Microsc 35:1151-1155.

Wilson CJ (2007) GABAergic inhibition in the neostriatum. In: Progress in brain research. GABA and the basal ganglia: from molecules to systems (Tepper JM, Abercrombie ED, Bolam JP, eds), pp 91-110. Amsterdam: Elsevier.

Wilson CJ, Groves PM, Kitai ST, Linder JC (1983) Three-dimensional structure of dendritic spines in the rat neostriatum. J Neurosci 3:383-388.

Xu ZC, Wilson CJ, Emson PC (1991) Restoration of thalamostriatal projections in rat neostriatal grafts: an electron microscopic analysis. J Comp Neurol 303:22-34. 